\title{
Solar Panels as Tip Masses in Low Frequency Vibration Harvesters
}

\author{
Jie Wang ${ }^{1}$, Mostafa R. A. Nabawy ${ }^{1,2, * \mathbb{C}}$, Andrea Cioncolini ${ }^{1}{ }^{1(\mathbb{D}}$ and Alistair Revell ${ }^{1}$ \\ 1 Department of Mechanical, Aerospace and Civil Engineering, The University of Manchester, \\ Manchester M1 3BB, UK; jie.wang-2@manchester.ac.uk (J.W.); andrea.cioncolini@manchester.ac.uk (A.C.); \\ alistair.revell@manchester.ac.uk (A.R.) \\ 2 Aerospace Engineering Department, Faculty of Engineering, Cairo University, Giza 12613, Egypt \\ * Correspondence: mostafa.ahmednabawy@manchester.ac.uk
}

Received: 13 September 2019; Accepted: 7 October 2019; Published: 9 October 2019

check for updates

\begin{abstract}
Tip masses are used in cantilevered piezoelectric energy harvesters to shift device resonance towards the required frequency for harvesting and to improve the electric power generation. Tip masses are typically in the form of concentrated passive weights. The aim of this study is to assess the inclusion of solar panels as active tip masses on the dynamics and power generation performance of cantilevered PVDF (polyvinylidene fluoride)-based vibration energy harvesters. Four different harvester geometries with and without solar panels are realized using off-the-shelf components. Our experimental results show that the flexible solar panels considered in this study are capable of reducing resonance frequency by up to $14 \%$ and increasing the PVDF power generated by up to $54 \%$. Two analytical models are developed to investigate this concept; employing both an equivalent concentrated tip mass to represent the case of flexible solar panels and a distributed tip mass to represent rigid panels. Good prediction agreement with experimental results is achieved with an average error in peak power of less than 5\% for the cases considered. The models are also used to identify optimum tip mass configurations. For the flexible solar panel model, it is found that the highest PVDF power output is produced when the length of solar panels is two thirds of the total length. On the other hand, results from the rigid solar panel model show that the optimum length of solar panels increases with the relative tip mass ratio, approaching an asymptotic value of half of the total length as the relative tip mass ratio increases significantly.
\end{abstract}

Keywords: vibration energy harvester; tip mass; PVDF; solar panels; low frequency; dynamic response; output power

\section{Introduction}

In the modern omni-connected world of Big-Data and the Internet of Things, there is an intense need for the provision of power to small wireless electronic devices. Applications include environmental sensing, equipment and process monitoring, and smart city applications-most of which require robust long-duration operation in remote and sometimes harsh environmental conditions. This demand has led to an increasing interest in developing energy harvesting solutions that would act as reliable, affordable, and environmentally friendly power sources for these devices and sensors. For such applications, piezoelectric devices are recognized as one of the most promising harvesting solutions. Indeed, there has been a significant amount of theoretical and experimental research to understand the dynamics and power generation characteristics for different configurations of piezoelectric harvesters, and among those cantilevered harvesters were probably the most studied configurations.

Piezoelectric energy harvesters can be realized from a range of materials; however, most efforts have considered ceramic (PZT (lead zirconate titanate)) or polymer (PVDF) materials with the former 
being the most studied configuration for base excitations (e.g., [1-5]) whereas the latter was the most studied for wind excitations (e.g., [6-11]). Other studies focused on comparing the performance of PZT- and PVDF-based harvesters when subjected to different sources of excitations. Considering wind and rain drop excitations, Vatansever et al. [12] assessed the effect of material dimensions, wind speed, drop mass, and releasing height of the drops on the amount of harvested power for polymer (using PVDF, part numbers: LDT1-028K and LDT4-028K, Measurement Specialties Inc. (MEAS), Hampton, VA, USA) and ceramic (single layer and bimorph PZTs) based devices. For excitations from rain drop impact, they showed that the PVDF LDT1-028K was by far generating the highest voltages. For wind excitation, they measured a power density value for the PVDF LDT4-028K of $157.9 \mu \mathrm{W} / \mathrm{cm}^{3}$ at $10 \mathrm{~m} / \mathrm{s}$ wind speed whereas ceramic-based harvesters showed less power density values of $9.67 \mu \mathrm{W} / \mathrm{cm}^{3}$ for the single layer configuration and $2.28 \mu \mathrm{W} / \mathrm{cm}^{3}$ for the bimorph configuration when tested at the same wind speed. Using an array of inline back-to-back cantilevered piezoelectric harvesters, Hobeck and Inman [13] compared the performance of these arrays when realized with PVDFs (LDT2-028K/L) versus when realized with PZT QuickPacks (model QP16n, Mide Technology Corp., Woburn, MA, USA). They demonstrated the superiority of the PZT harvester array showing its capability to achieve $1 \mathrm{~mW}$ per cantilever at a mean wind speed of $11.5 \mathrm{~m} / \mathrm{s}$ whereas the PVDF harvester array achieved $1.2 \mu \mathrm{W}$ per cantilever at wind speed of $7 \mathrm{~m} / \mathrm{s}$.

The existing literature clearly indicates that each of the PZT and PVDF options has its strengths and weaknesses. PZT-based harvesters have relatively higher electromechanical coupling coefficient, higher mechanical quality factors, and larger stiffness; however, they also possess some serious disadvantages such as having a heavy content of lead which is a serious environmental hazard [14]. PZT-based harvesters are also fragile, only limited to small deformations, and have high economic cost. On the other hand, and despite of the relatively low electromechanical coupling coefficients, PVDF-based harvesters are environmentally friendly, allow large deformations, have greater resistance to mechanical shocks, and are much lower in their economic cost [14,15]. Nevertheless, the main advantage of PVDF-based energy harvesters has been their superiority in scavenging mechanical energy at low frequencies leading to an increased interest over the recent years to study their performance within such category of energy harvesting applications [14-19].

An energy harvesting device is likely to be small in comparison to traditional power generation technologies, and as such achieving relevant power output is important. An interesting strategy, therefore, is to consider the harvesting of multiple ambient energies within the same device at the same time [20]. This approach has shown success in simultaneously harvesting wind and wave energies [21], wind and structural vibration energies [22], and wind and solar energies [9-11]. Gambier et al. [23] considered a cantilevered structure capable of harvesting energies from base acceleration, solar, and thermal excitations. They developed a multilayer cantilever with piezoceramic, thin-film battery, and metallic substructure layers. This cantilever was then bonded and fully covered with flexible solar panels. Jiang et al. [15] proposed using a magnet as a tip mass for their PVDF-based cantilever harvester. A coil was located below this magnetic tip allowing simultaneous electromagnetic energy harvesting. These particular studies $[15,23]$ provided the motivation for the current work: to develop PVDF energy harvesting cantilevers for low frequency vibration applications that use functional tip masses rather than the traditionally used passive tip masses. To do so, we decided to understand the feasibility/benefit of using solar panels to act as these functional tip masses.

The primary benefit of attaching a solar panel to the free end of a cantilever beam piezoelectric harvester is clearly the generation of power from solar energy. This concept has been demonstrated in preliminary work by the authors [9-11] for the case of wind excitation. However, the simultaneous harvesting of energy from base vibration and light sources within a PVDF-based harvester has not been considered. The objective of the present work is, therefore, to investigate the extent to which the additional mass of the panels can be exploited to favorably modulate the dynamics of the harvester and therefore improve its piezoelectric power generation. The effect of varying the geometry (length and width) of these harvesters is also investigated in this study. We constructed eight harvesters of varying 
dimensions using commercial-off-the-shelf components and experimentally assessed their dynamics and power generation characteristics. Following experimental testing we developed two different analytical models of this configuration; the first employs a concentrated tip mass representation whereas the second employs a distributed tip mass representation. We compared models to our experiments and against each other, then applied them to explore and assess the different possible tip mass configurations to provide a fast evaluation of the optimal design for the harvesters considered in this work.

\section{Experimental Set-up}

\subsection{Harvesters Design and Realisation}

A schematic diagram of the harvester design is provided in Figure 1, together with images of the eight harvesters realized and tested here. The harvesters have been constructed using a stainless-steel shim core of $0.1 \mathrm{~mm}$ thickness by Precision Brand Products, Inc. (Downers Grove, IL, USA (www.precisionbrand.com); density, $\rho_{\mathrm{e}}=7900 \mathrm{~kg} / \mathrm{m}^{3}$; Young's modulus, $Y_{\mathrm{e}}=180 \mathrm{GPa}$ ) which has been covered on both sides with a layer of flexible PVDF piezoelectric elements in a sandwich arrangement. The PVDF elements used are the LDT2-028K model from TE Connectivity Ltd. (Schaffhausen, Switzerland (www.te.com); density, $\rho_{p}=1780 \mathrm{~kg} / \mathrm{m}^{3}$; Young's modulus, $Y_{p}=2.3 \mathrm{GPa}$; piezo strain constant, $d_{31}=23 \times 10^{-12} \mathrm{C} / \mathrm{N}$; capacitance, $C=2.85 \mathrm{nF}$ ). Note that each PVDF element comprises a PVDF film covered with silver ink screen printed electrodes, all contained within a thin plastic coating for protection. The PVDF elements were attached to the metal shim with small pieces of thin tape to ensure that the PVDF elements deflection followed closely that of the metal shim. Each harvester was visually inspected to ensure that PVDF elements were fully attached to the metal shim and closely followed its motion, without bulging or deformation during deflection. Only a few $\mathrm{cm}^{2}$ of tape were used for each harvester, so that the added mass of the tape can be neglected, as well as any other effect of the tape on the mechanical response of harvesters.

The dimensions of one PVDF element are $73 \mathrm{~mm} \times 16 \mathrm{~mm} \times 0.2 \mathrm{~mm}$ (total length $\times$ width $\times$ thickness); however, the overhang length of the harvester from the fixed support to the free tip was varied through changing the clamp position. For example, harvesters H1 and H2 in Figure 1 are nominally identical but differ in the overhang length due to the position of the clamp. Similarly for the harvesters $\mathrm{H} 3-\mathrm{H} 4, \mathrm{H} 5-\mathrm{H} 6$, and H7-H8. Varying the overhang length of the harvester via different clamp positions is an effective way to change the active length of the PVDF elements whilst using off-the-shelf components that come in predetermined size. The portion of the PVDF element enclosed within the clamp, in fact, does not deform and therefore does not produce any power. In particular, we tested two different overhang lengths and two different widths, resulting in a total of four geometric configurations for the harvesters (H1 through $\mathrm{H} 4$ in Figure 1d). The use of off-the-shelf PVDF elements gave more freedom in modulating the harvester length as compared to its width, which was constrained to be a multiple (4 or 7 in the present case) of the width of a single PVDF element.

For each of the baseline geometries employed in this work (H1 through H4 in Figure 1d), a corresponding version with two mini flexible solar panels attached at the tip was also considered, one on each side as shown in Figure $1 \mathrm{~b}$ and H5 through H8 in Figure 1e, thus resulting in a total of eight harvesters realized and tested. The flexible solar panels used were manufactured by PowerFilm, Inc. (Ames, IA, USA (www.powerfilmsolar.com); density $\rho_{s}=1250 \mathrm{~kg} / \mathrm{m}^{3}$ ). Two models were used: the first is the SP3-37 capable of generating $22 \mathrm{~mA} @ 3 \mathrm{~V}$, and has dimensions of $64 \mathrm{~mm} \times 36.8 \mathrm{~mm} \times$ $0.22 \mathrm{~mm}$ (width $\times$ length $\times$ thickness), whereas the second is the MP3-37 that is capable of generating $50 \mathrm{~mA} @ 3 \mathrm{~V}$, and has dimensions of $112 \mathrm{~mm} \times 36.8 \mathrm{~mm} \times 0.22 \mathrm{~mm}$. As evident in Figure 1, the size of the solar panels was selected to fit into the present harvesters. The main geometrical details of the present harvesters are summarized in Table 1. 


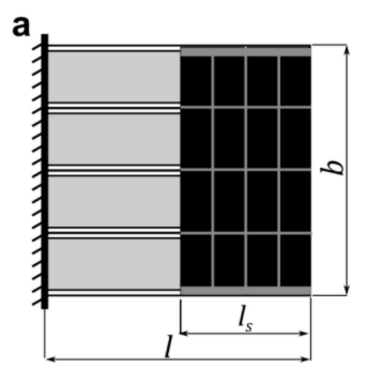

b

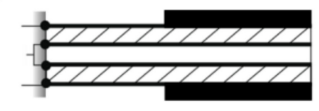

elastic layer PVDF layer $\triangle \backslash \backslash \bar{z} h_{p}$ solar layer
C

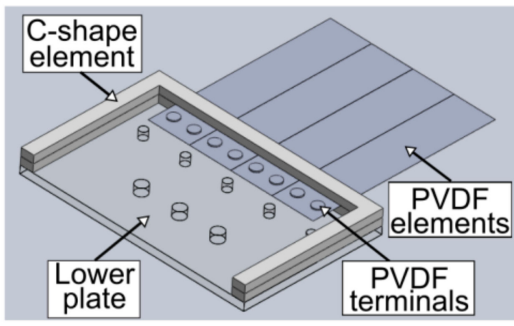

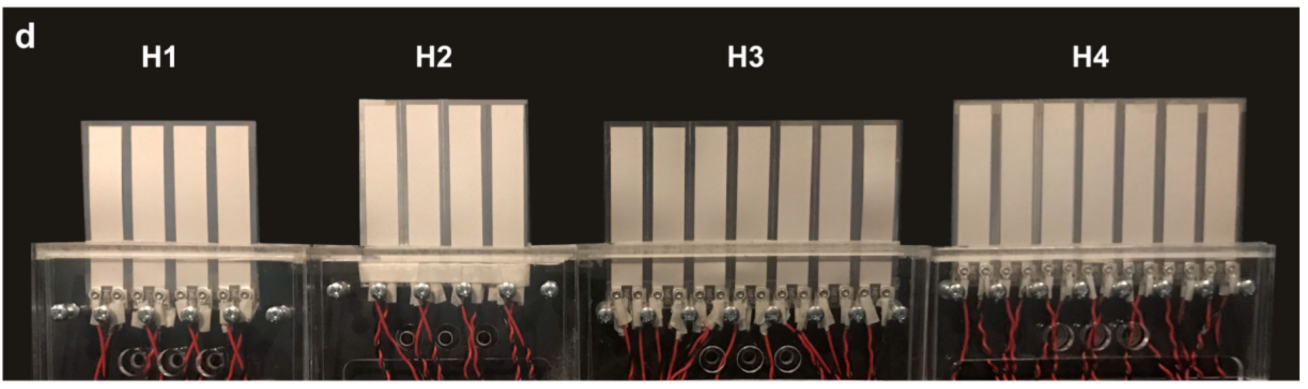

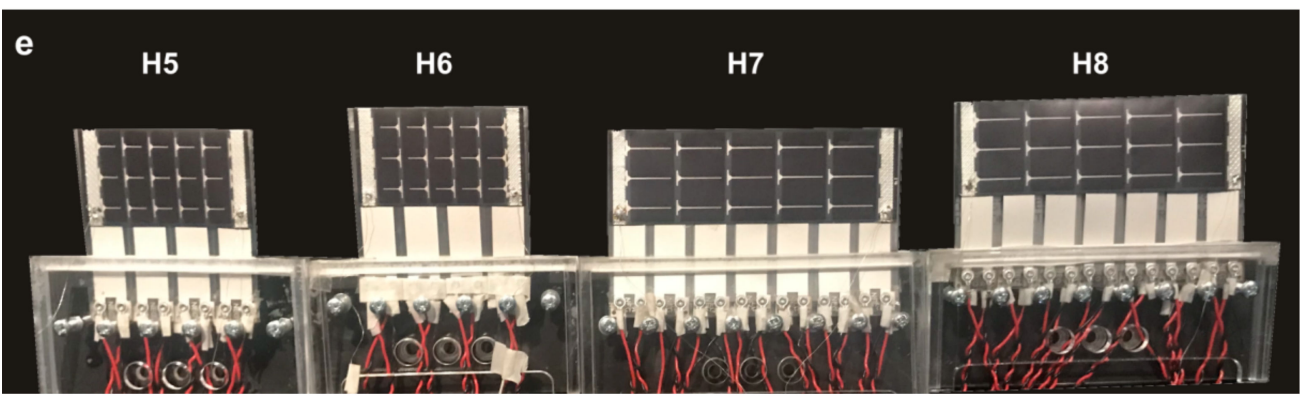

Figure 1. The configuration of the harvesters considered in this study: (a) and (b) schematics of the harvester construction; (c) schematic representation of the custom-made mounting system to connect the harvester to the shaker; (d) four harvesters realized without incorporating solar panels; (e) four harvesters with solar panels.

Table 1. Geometric characteristics of the harvesters considered in this study.

\begin{tabular}{ccccccc}
\hline \multirow{2}{*}{ Harvester } & No of PVDFs & Solar Panel & Length $(\mathbf{m m})$ & Width $(\mathbf{m m})$ & \multicolumn{2}{c}{ Beam aspect ratio } \\
\cline { 5 - 7 } & & & & $\frac{l}{\left(h_{e}+2 h_{p}\right)}$ & $\frac{l-l_{s}}{\left(h_{e}+2 h_{p}\right)}$ \\
\hline H1 & $8(4 /$ side $)$ & NA & 48 & 64 & 96 & NA \\
H2 & $8(4 /$ side $)$ & NA & 58 & 64 & 116 & NA \\
H3 & $14(7 /$ side $)$ & NA & 48 & 112 & 96 & NA \\
H4 & $14(7 /$ side $)$ & NA & 58 & 112 & 116 & NA \\
H5 & $8(4 /$ side $)$ & SP3-37 & 48 & 64 & 96 & 22.4 \\
H6 & $8(4 /$ side $)$ & SP3-37 & 58 & 112 & 96 & 42.4 \\
H7 & $14(7 /$ side $)$ & MP3-37 & 48 & 112 & 116 & 22.4 \\
H8 & $14(7 /$ side $)$ & MP3-37 & 58 & & 42.4 \\
\hline
\end{tabular}

The harvesters were connected to the test apparatus with a custom-made mounting system that was manufactured from laser cut acrylic, as shown schematically in Figure 1c. The mounting system was designed to provide a cantilever boundary condition at the harvester base by including C-shape elements to sit between the mounting upper/lower plates and the harvester. This avoids any direct contact between the mounting system and the PVDF terminals, while providing a very strong fixation.

It may be worth reiterating that the main objective of the present work was to assess the feasibility of using solar panels as functional tip masses on PVDF-based piezoelectric harvesters, and hence was carried out relying entirely on available off-the-shelf components for the metal shim, the PVDF elements, and the solar panels used to realize the harvesters. This posed limitations on the dimensions 
of the harvesters that could be realized and explored. In particular, the width of available solar panels that could allow full coverage of multiple PVDF elements was the main driver in selecting these two models of solar panel, and hence the two values of the harvester width investigated in this study. The possibility of having the metal shim, the PVDF elements, and the solar panels custom-made to specific desired dimensions and material properties will be exploited in a future optimization stage to better explore the parameter space of the harvesters and to tailor their mechanical properties to the intended applications, but is not further considered at this stage.

\subsection{Harvesters Identification}

For the purposes of this study, it is essential to identify the mechanical and electrical characteristics of the harvesters considered, which include: (1) the equivalent tip mass of the solar panels; (2) the natural frequency of the harvester; (3) the damping ratio of the harvester; (4) the stiffness of the harvester; and (5) the optimum load resistances for the PVDF and solar elements that maximize the generated electrical power.

As evident in Figure 1, the solar panels span over the harvester tip region, and are not actual concentrated tip masses. Here we propose to transform the distributed mass of the solar panels into an equivalent concentrated mass located at the tip of the harvester. This was achieved by first assuming that the solar panels are homogeneous with their center of gravity located right at the center of the panel. In the case of small angular displacements of the harvester (which is consistent with the small base excitation acceleration level used in this study), Rao [24] has shown that an equivalent concentrated tip mass can be estimated by equating the kinetic energy of the actual mass (in our case the solar panels) to that of an equivalent mass located at the tip of the harvester. Applying this approach to our configuration led to the following expression for the equivalent concentrated tip mass, $m_{\text {tip }, e q}$ :

$$
m_{\text {tip }, e q}=\rho A_{s} l_{s}\left(\frac{l-\left(\frac{l_{s}}{2}\right)}{l}\right)^{2}=2 \rho_{s} h_{s} b l_{s}\left(\frac{l-\left(\frac{l_{s}}{2}\right)}{l}\right)^{2}
$$

where $\rho A_{s}$ is the mass per unit length of the solar panels, $\rho_{s}$ is the density of the solar panel, $l_{s}$ is the length of the solar panel, $l$ is the overhang length of the harvester, $b$ is the width of the harvester/solar panel, and $h_{s}$ is the thickness of the solar panel. The factor two in the above equation is to account for having two solar panels, one at each side of the harvester. Note that the squared bracket in Equation (1) could be seen as a correction factor through which the solar panels mass has been converted to an "equivalent concentrated" mass at the tip. Whilst this approach is simple, particularly when compared with the more complex approaches for dealing with distributed masses in the literature (e.g., [25-27]), it was found to be sufficiently effective in capturing the behavior of the thin, flexible solar panels employed in this study as will be shown later in Section 3.2. Following Lord Rayleigh [28], the equivalent mass of the whole harvester, $M_{e q}$ is thus evaluated from:

$$
M_{e q}=\frac{33}{140} m_{\text {harv }}+m_{\text {tip }, e q}
$$

where $m_{\text {harv }}$ is the structural mass of the harvester given by:

$$
m_{\text {harv }}=\rho A_{\text {harv }} l=\left(\rho_{e} h_{e}+2 \rho_{p} h_{p}\right) b l
$$

where $\rho A_{\text {haro }}$ is the mass per unit length of the harvester, $\rho$ denotes density, and $h$ denotes thickness, whereas the subscripts $e$ and $p$ represent the elastic metal layer and the PVDF active layer, respectively; the factor two in the second term in brackets accounts for having two layers of PVDFs, one at each side of the metal shim.

Natural frequencies and damping ratios of the harvesters were experimentally measured through preliminary free-decay vibration tests. With the harvesters clamped in a cantilever configuration, 
the harvester tip was manually deflected of about $10^{\circ}-20^{\circ}$ from its rest position, which is small enough to trigger a mode- 1 free-vibration, and then released. The oscillation of the harvester tip was tracked using a laser vibrometer (PDV-100, Polytec $\mathrm{GmbH}$, Waldbronn, Germany (www.polytec.com)) providing displacement time-series of the harvester free-end. The natural frequency was then determined from the period between peaks on the free-decay amplitude, whereas the damping ratio was determined from the logarithmic decrement of the free-decay amplitudes of motion. Once the mode- 1 natural frequency, $\omega_{1}$ and equivalent mass, $M_{e q}$ of the harvester were determined, the harvester's stiffness, $K$ was then estimated using the classical equation [24]:

$$
\omega_{1}=2 \pi f_{1}=\sqrt{\frac{K}{M_{e q}}}
$$

Table 2 provides the identified values of natural frequency, damping ratio and stiffness for the eight harvesters considered in this work.

Table 2. Mechanical and electric properties of the harvesters considered in this study.

\begin{tabular}{|c|c|c|c|c|c|c|c|c|}
\hline Harvester & H1 & $\mathrm{H} 2$ & H3 & H4 & H5 & H6 & H7 & H8 \\
\hline Natural frequency, $f_{1}(\mathrm{~Hz})$ & 43.5 & 27.0 & 43.5 & 26.3 & 39.5 & 23.8 & 38.5 & 22.7 \\
\hline Damping ratio, $\zeta_{1}$ & 0.057 & 0.056 & 0.051 & 0.066 & 0.049 & 0.058 & 0.047 & 0.066 \\
\hline Stiffness, $K(\mathrm{~N} / \mathrm{m})$ & 81.17 & 37.88 & 142.1 & 62.87 & 97.68 & 42.93 & 161.5 & 68.46 \\
\hline Optimum load resistance for PVDFs, $R_{\text {opt }}(\mathrm{kOhm})$ & 161 & 258 & 92 & 152 & 179 & 293 & 104 & 176 \\
\hline
\end{tabular}

Optimum load resistances that allowed a peak electric power generation were separately determined for the PVDF elements and the solar panels. For the PVDF elements, theoretical values of the optimum load resistance at the harvester first resonance frequency were evaluated using the analytical expression [25,29]:

$$
R_{o p t}=\frac{1}{\omega_{1} C_{e q}}\left(\frac{2 \zeta_{1}}{\sqrt{4 \zeta_{1}^{2}+k_{31}^{4}}}\right)
$$

where $R_{o p t}$ is the optimum load resistance value and $C_{e q}$ is the equivalent internal capacitance for the PVDF elements. In the present harvester design, the PVDF elements are connected in parallel, hence the equivalent capacitance, $C_{e q}$ is simply the internal capacitance of each PVDF element $(2.85 \mathrm{nF})$ multiplied by the number of PVDF elements included in the harvester. Moreover, since the electromechanical coupling coefficient of the PVDF elements $k_{31}$ is low $(12 \%,[15,30])$, the bracketed term in Equation (5) is almost unity. Values of the optimum theoretical resistances from Equation (5) for all harvesters are provided in Table 2. To ensure that Equation (5) is valid for the current configuration, the optimum load resistance was also empirically determined. The optimum load resistance values identified experimentally were very close to those determined from Equation (5), with an average difference on the order of $\pm 5-10 \%$. As such, for simplicity and consistency, the identified theoretical values from Equation (5) were used as an acceptable representation of the optimum load resistance values for the PVDFs.

For the solar panels, the optimum load resistance was determined empirically using a variable load that was tuned to maximize the power output. The solar panels were illuminated using a light-emitting diode (LED) light (Capra 12 Daylight, Vibesta, Helmond, The Netherlands (www.vibesta.com)) that was located perpendicular to the surface of the harvester. Other indoor light sources were not considered at this stage. The lux illumination from the LED at the position of the solar panel was measured with a portable light meter (DT-1309 USB Logging Light Meter, ATP Instrumentation Ltd, Ashby-de-la-Zouch, UK (www.atp-instrumentation.co.uk)). Then, the optimum load resistance value that would allow a peak electric power generation was identified (note that the measured power values will be provided 
later in Section 6). Table 3 provides the experimental optimum load resistance values measured for the two models of solar panels adopted in this study.

Table 3. Optimum load resistance values for solar panels.

\begin{tabular}{ccc}
\hline \multirow{2}{*}{ Lux } & \multicolumn{2}{c}{ Resistance (kOhm) } \\
\cline { 2 - 3 } & PowerFilm SP3-37 & PowerFilm MP3-37 \\
\hline 500 & 15.4 & 2.82 \\
1000 & 8.80 & 1.54 \\
1500 & 7.00 & 1.10 \\
2000 & 5.00 & 0.88 \\
2500 & 4.70 & 0.65 \\
3000 & 3.76 & 0.57 \\
3500 & 2.82 & 0.49 \\
4000 & 2.82 & 0.49 \\
4500 & 2.35 & 0.41 \\
5000 & 2.35 & 0.41 \\
\hline
\end{tabular}

\subsection{Experimental Apparatus}

The experimental set-up used to investigate the performance of the present harvesters is shown in Figure 2. The set-up includes a shaker (V55, Data Physics, San Jose, CA, USA (www.dataphysics.com)) with control signals provided by a signal generator (AFG1022, Tektronix Inc., Beaverton, OR, USA (www.tek.com)) operated in sine wave mode. The input voltage amplitude driving the shaker was tuned to yield a base acceleration value of $0.5 \mathrm{~g}$, which was not varied during the tests. Two reasons were behind the selection of this acceleration amplitude. First, $0.5 \mathrm{~g}$ is within the range of acceleration magnitudes for various vibration sources, as reported in [29], and is therefore representative of actual harvesting applications. Second, an acceleration of $0.5 \mathrm{~g}$ is small enough to model the harvesters as linear electromechanical systems, thus avoiding piezo-elastic, dissipation, and geometric non-linearities [31]. The base excitation level was measured using an accelerometer (PCB 336M13, PCB Piezotronics, Depew, NY, USA (www.pcb.com)) attached and secured to the shaker. For each experimental run, the harvester tip motion was recorded via a laser vibrometer (PDV-100, Polytec).

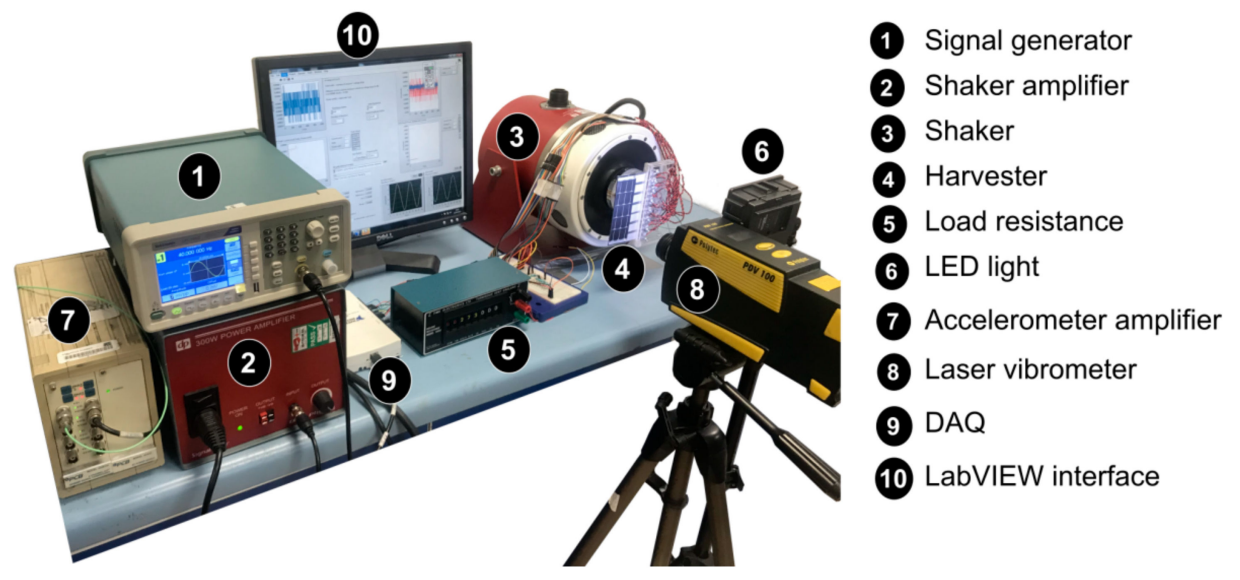

Figure 2. Experimental set-up used in the current study.

Each harvester was connected to the identified optimum load resistance. The electric power generated from the harvesters was collected and processed through LabVIEW (LabVIEW 2017, National Instruments, Austin, TX, USA (www.ni.com)) and the data was gathered through an external data acquisition (DAQ) device (NI-USB-6225, National Instruments, Austin, TX, USA (www.ni.com)). The data acquisition program was written as a Virtual Instrument (VI) in LabVIEW 2017 using the 
standard DAQ-mx library. This program gathered, saved and displayed the data with some processing to allow an immediate impression of the power generated to be seen and to reaffirm that the load resistance is allowing the highest power output. The sampling rate was set at $1 \mathrm{kHz}$ to allow sufficient resolution of data through a vibration cycle.

\section{Considerations for Harvesters with Flexible Solar Panels}

\subsection{Model for an Equivalent Concentrated Tip Mass}

Piezoelectric cantilever beams have always been attractive systems for analytical modelling (e.g., see [1,2,25-27,29-33]). For harvesting applications, both lumped parameter and distributed parameter models have been considered. However, lumped parameter models typically need a correction factor to improve their prediction capabilities when compared to distributed parameter models (for a comprehensive analysis of this point, the reader is referred to [31]). As such, distributed parameter models are adopted in this study. For simplicity it was decided that PVDF elements could be represented as continuous media in the span-wise direction without significant loss of accuracy, such that a typical bimorph model could be employed to represent two piezoelectric active layers sandwiching an elastic passive layer [2,31,32]. Validation results in Section 3.2 support our hypothesis that this assumption does not significantly impact the model ability to predict the general dynamics of the device.

Distributed parameter models based on the Euler-Bernoulli beam theory are used, implying a thin beam assumption [34] which is known to be acceptable for the length to thickness ratios used in the current work, which were in excess of 20 (see Table 1). In this section, we consider a first modelling approach where the solar panels are represented as an equivalent concentrated tip mass (evaluated based on Equation (1)), and hence are of negligible mass moment of inertia about the tip, see Figure 3 . This approach, thus, assumes that the solar panels do not restrain the deformation of the beam section they cover. As such, this approach better represents thin, flexible panels that do not significantly alter the beam deformation pattern as is the case for those employed in the experimental part of this study.
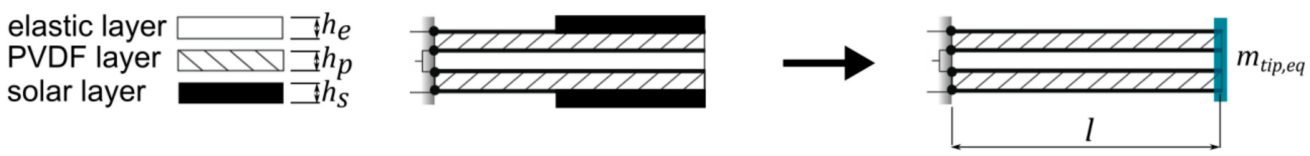

Figure 3. First modelling approach used in the current study where solar panels are represented as an equivalent concentrated tip mass. Equivalent tip mass is highlighted in blue.

The characteristic equation for a distributed parameter model of an Euler-Bernoulli beam with a concentrated tip mass as that shown in Figure 3 is known to be [31]:

$$
1+\cos \lambda_{n} \cosh \lambda_{n}+\lambda_{n} \mu\left(\cos \lambda_{n} \sinh \lambda_{n}-\sin \lambda_{n} \cosh \lambda_{n}\right)=0
$$

where $\mu=\frac{m_{\text {tipeq }}}{m_{\text {harv }}}$ with $m_{\text {tip }, e q}$ and $m_{\text {harv }}$ being evaluated based on Equations (1) and (3). Hence Equation (6) may be solved for the dimensionless $n^{\text {th }}$ eigenvalue, $\lambda_{n}$. The eigen function (mode shape) can be obtained as [31]:

$$
X_{n}(x)=A_{n}\left[\cos \lambda_{n} \frac{x}{l}-\cosh \lambda_{n} \frac{x}{l}+\sigma_{n}\left[\sin \lambda_{n} \frac{x}{l}-\sinh \lambda_{n} \frac{x}{l}\right]\right]
$$

where $\sigma_{n}$ is obtained from:

$$
\sigma_{n}=\frac{\sin \lambda_{n}-\sinh \lambda_{n}+\lambda_{n} \mu\left(\cos \lambda_{n}-\cosh \lambda_{n}\right)}{\cos \lambda_{n}+\cosh \lambda_{n}-\lambda_{n} \mu\left(\sin \lambda_{n}-\sinh \lambda_{n}\right)}
$$


and $A_{n}$ is the modal amplitude which can be obtained making use of any of the orthogonality conditions such as:

$$
\int_{0}^{l} X_{S}(x) b\left(\rho_{e} h_{e}+2 \rho_{p} h_{p}\right) X_{r}(x) d x+X_{s}(l) m_{\text {tip }, e q} X_{r}(l)=\delta_{r s}
$$

where $\delta_{r s}$ is the Kronecker delta. The displacement of the harvester is thus obtained from:

$$
w(x, t)=\sum_{n=1}^{\infty} \psi_{n} X_{n}(x) \frac{1}{\omega_{n}^{2} \sqrt{\left(1-\left(\frac{\omega}{\omega_{n}}\right)^{2}\right)^{2}+\left(2 \zeta_{n} \frac{\omega}{\omega_{n}}\right)^{2}}} \mathrm{~A}_{b} \cos \omega t
$$

where $w$ is the displacement along the beam length relative to the base, $\omega$ is the operation angular frequency, $\mathrm{A}_{b}$ is the base excitation acceleration amplitude, and $t$ denotes time. The expression for $\psi_{n}$ is:

$$
\psi_{n}=b\left(\rho_{e} h_{e}+2 \rho_{p} h_{p}\right) \int_{0}^{l} X_{n}(x) d x+m_{t i p, e q} X_{n}(l)
$$

Clearly, the interest is in motion around the first mode as well as in the harvester's tip displacement as it allows maximum displacement amplitude; as such, Equation (10) becomes:

$$
w(l, t)=\psi_{1} X_{1}(l) \frac{1}{\omega_{1}^{2} \sqrt{\left(1-\left(\frac{\omega}{\omega_{1}}\right)^{2}\right)^{2}+\left(2 \zeta_{1} \frac{\omega}{\omega_{1}}\right)^{2}}} A_{b} \cos \omega t
$$

and hence the tip displacement steady state frequency response transmissibility can be expressed as:

$$
\left|H_{\text {disp }}\right|=\frac{w(l)}{A_{b}}=\psi_{1} X_{1}(l) \frac{1}{\omega_{1}^{2} \sqrt{\left(1-\left(\frac{\omega}{\omega_{1}}\right)^{2}\right)^{2}+\left(2 \zeta_{1} \frac{\omega}{\omega_{1}}\right)^{2}}}
$$

The model employed to evaluate the parallel connection steady state voltage response, $v_{p}$ is based on the bimorph model $[2,31]$ as:

$$
\frac{v_{p}}{w(x)}=\left|\frac{j\left(\omega R_{o p t} \kappa_{n}\right)}{\left(1+j \omega R_{o p t} C_{p}\right) X_{n}(x)}\right|
$$

where $C_{p}$ is the internal capacitance given by:

$$
C_{p}=\frac{2 \varepsilon b_{p} l}{h_{p}}
$$

where $b_{p}$ is the effective width of the PVDF layer which in our case is the number of PVDF elements on one side of the harvester multiplied by the effective width of the active part of each element $(12 \mathrm{~mm}$ for the LDT2-028K model). The term $\kappa_{n}$ is the modal coupling term which for a bimorph configuration is given by:

$$
\kappa_{n}=2 \frac{d_{31} Y_{p} b_{p}}{2}\left(h_{p}+h_{e}\right) \int_{0}^{l} \frac{d^{2} X_{n}(x)}{d x^{2}} d x
$$

We are most interested in the peak steady state voltage as this allows the peak power generation. This occurs when operating at the first resonance frequency; hence Equation (14) becomes:

$$
v_{p, \max }=\left|\frac{j\left(\omega_{1} R_{o p t} \kappa_{1}\right)}{\left(1+j \omega_{1} R_{o p t} C_{p}\right) X_{1}(l)}\right| w(l)
$$


where $X_{1}(l)$ is evaluated based on the expression provided in Equation (7). The root mean square value of the peak power is thus evaluated from:

$$
P_{\max }=\frac{v_{p, \max }^{2}}{2 R_{\text {opt }}}
$$

\subsection{Equivalent Concentrated Tip Mass Model vs. Experimental Measurements}

Figure 4 provides a comparison between the experimentally measured tip displacement Frequency Response Function (FRF) and the predicted tip displacement using the equivalent concentrated tip mass model (for H1-4, tip mass was set to zero). Despite of its simplicity, it is evident that the model is capable of capturing the experimental results with good accuracy for the eight cases considered. Note that, all numerical modelling was undertaken using MATLAB (R2018a, MathWorks, Natick, MA, USA (www.mathworks.com)). The results in Figure 4 allow several observations: First, the harvester length has a clear influence on the tip displacement values: Longer harvesters ( $\mathrm{H} 2$ and $\mathrm{H} 4$ as well as $\mathrm{H} 6$ and H8) have larger tip displacement amplitude and larger ratio of tip displacement to beam length. Second, harvesters with solar panels deliver higher tip displacement values for all cases. These two observations have a significant effect on the generated power values as will be shown later. Third and as expected, Figure $4 \mathrm{a}$ confirms that the resonance frequency increases with decreasing the length, and that $\mathrm{H} 1$ and $\mathrm{H} 3$ as well as $\mathrm{H} 2$ and $\mathrm{H} 4$ have similar resonance frequency values. This is also evident from Table 2 where these pairs have almost the same resonance frequencies (with the note that if any tiny difference between the experimental resonance frequencies of these pairs exists, it is mainly due to minor manufacturing differences). This is expected as apart from the width these harvester pairs have the same exact configuration. Fourth, comparing Figure $4 a, b$, it is evident that the solar panels shift the resonance frequencies to lower values, hence are capable of modulating the operational frequency of the device. This reduction in the resonance frequency varied between 14\% (for $\mathrm{H} 4$ and $\mathrm{H} 8$ ) and 10\% (for $\mathrm{H} 1$ and $\mathrm{H} 5)$.

(a)

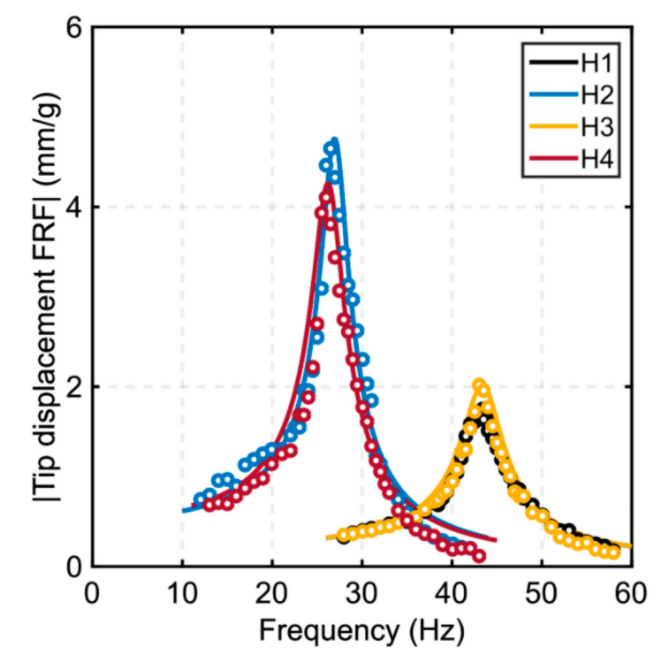

(b)

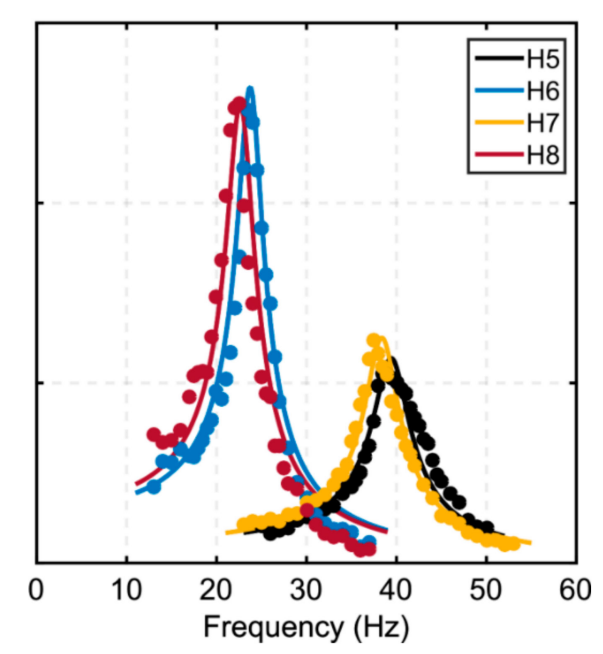

Figure 4. Measured and predicted tip displacement output FRFs for the eight harvesters. (a) Harvesters without solar panels (empty circle markers). (b) Harvesters with solar panels (filled circle markers). Markers represent the experimental measurements whereas solid lines represent the equivalent concentrated tip mass model predictions.

Figure 5 shows the PVDF power frequency response of the eight harvesters. Some observations are evident: First, the equivalent concentrated tip mass model employed is predicting the output PVDF power with good accuracy. Second, the PVDF power output from the longer harvesters is higher which 
is consistent with the tip displacement comparison discussed above. Third, the effect of the harvester width is more pronounced in the power results (as compared to displacement results) where it is clearly evident that wider harvesters are capable of generating more PVDF power. Finally, comparing Figure $5 a, b$, it is evident that harvesters with solar panels are capable of generating more PVDF power confirming the ability of the employed solar panels to act as effective tip masses. In fact, the increase in PVDF power due to the presence of solar panels could be up to $54 \%$ as when comparing $\mathrm{H} 1$ and $\mathrm{H} 5$.

(a)

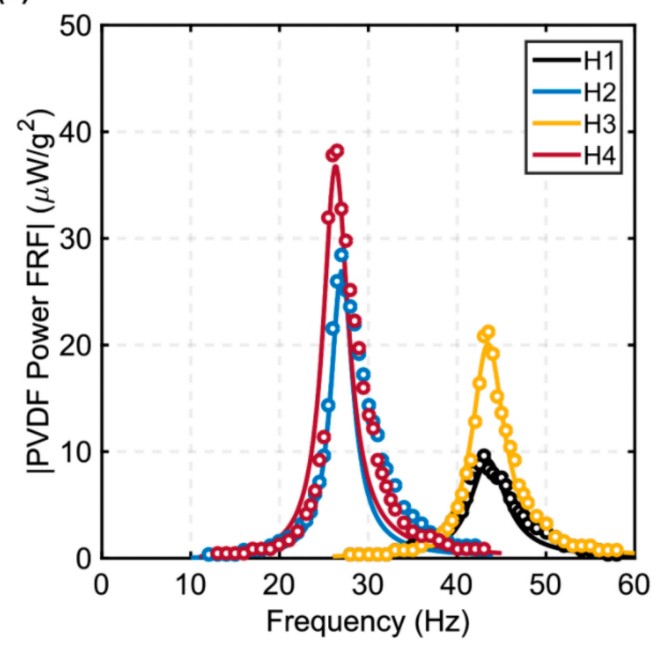

(b)

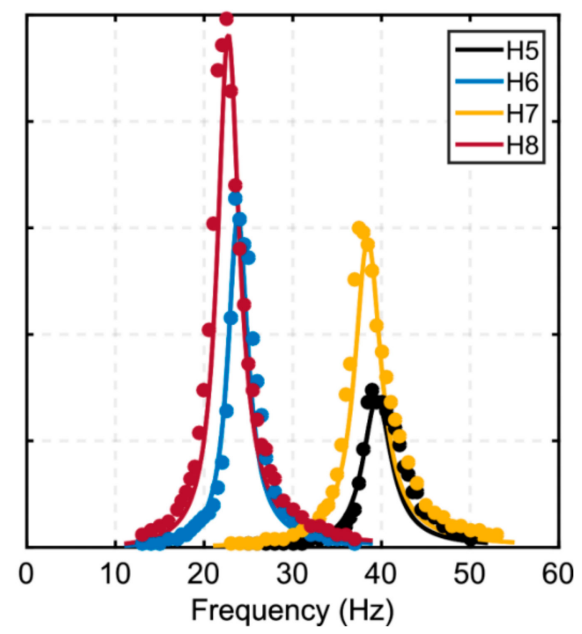

Figure 5. Measured and predicted power output FRFs for the PVDF elements of the eight harvesters. (a) Harvesters without solar panels (empty circle markers). (b) Harvesters with solar panels (filled circle markers). Markers represent the experimental measurements whereas solid lines represent equivalent concentrated tip mass model predictions.

In order to further assess the model capability in predicting the electric power generation, Figure $6 \mathrm{a}$ shows a comparison between the measured versus predicted PVDF peak power (i.e., PVDF power at resonance). Here we limit this assessment to the PVDF peak power as this is arguably the most important metric for assessment. To provide an estimate of accuracy in predicting PVDF peak power, Figure $6 \mathrm{~b}$ shows the error values between the equivalent concentrated tip mass model predictions and experimental measurements for the eight harvesters considered in this work. The model typically under-predict the measured PVDF peak power with a mean error for the eight harvesters of $-4.5 \%$. This discrepancy may be due to the assumptions adopted in the model, manufacturing imperfections, errors in experimental measurement, or a combination of these. Nevertheless, this discrepancy is small enough to be of minor concern for practical applications.

It is instructive to understand how the length and width affect performance. Note that, in the current study, we did not consider the effect of the PVDF elements thickness as this is constrained to what was available in the market. Figure 6a could be used to assess the variation of PVDF peak power for the different lengths and widths considered. For both harvester groups (with and without solar panels), the conclusion is the same: to achieve higher PVDF peak power values, the harvester should be longer and wider. Moreover, it could be seen that the length is more influential in achieving higher power values compared to width. 
(a)

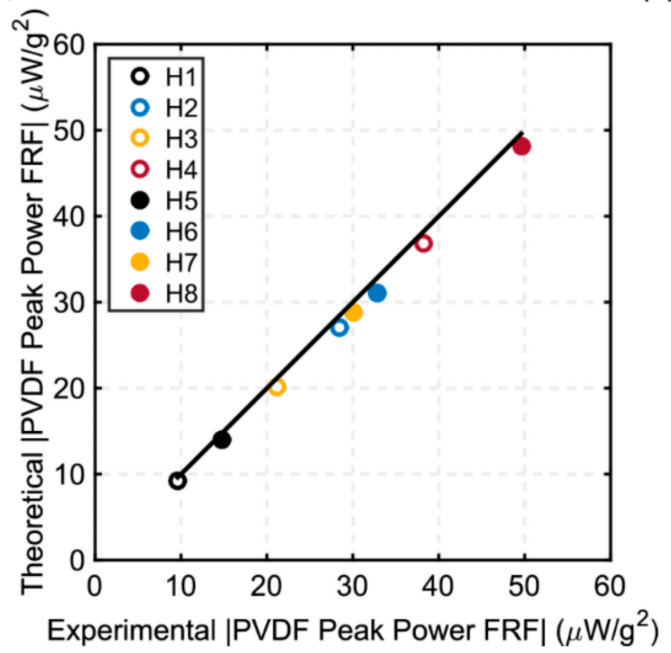

(b)

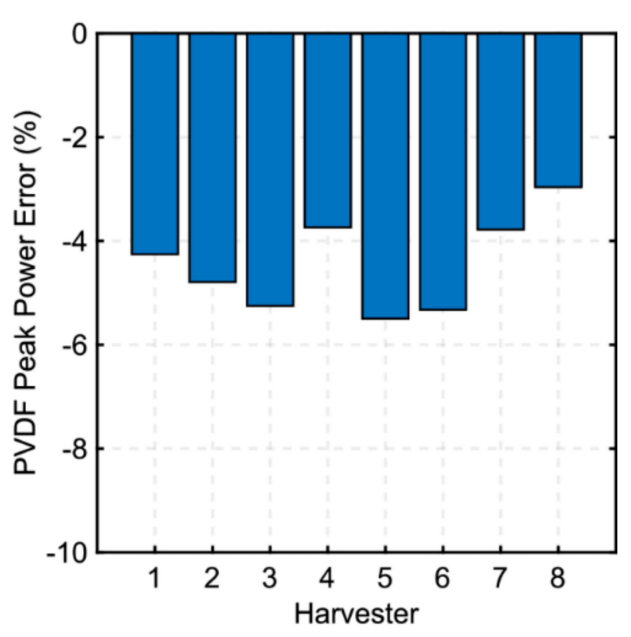

Figure 6. Comparison between experimental and equivalent concentrated tip mass model results. (a) Measured vs predicted PVDF peak power of the eight harvesters. (b) Prediction error for PVDF peak power as compared to experimental measurements.

\section{Considerations for Harvesters with Rigid Solar Panels}

\subsection{Model for a Distributed Tip Mass}

In the previous section the good agreement between experiment and the simple analytical model demonstrated that the presence of solar panels in our lab experiment may be considered to have a negligible influence on the deformation, since they are light and flexible. While our tests are in general limited to the material available from distributors, it is important to consider how our findings might hold when harvesters are scaled-up or tuned to different dynamics. In this section, we consider the scenario when the solar panels have a significant rigidity which acts to reduce the deformation of the beam. Given that the piezoelectric layer must deform to generate electricity it is important to consider the case where the presence of the solar panels prevents deformation at the tip region. Accordingly, a model is developed where the solar panels and the beam section they cover are represented as a rigid distributed tip mass as shown in Figure 7. This implies that the solar panel entirely prevents the deformation of the beam for the section it covers.
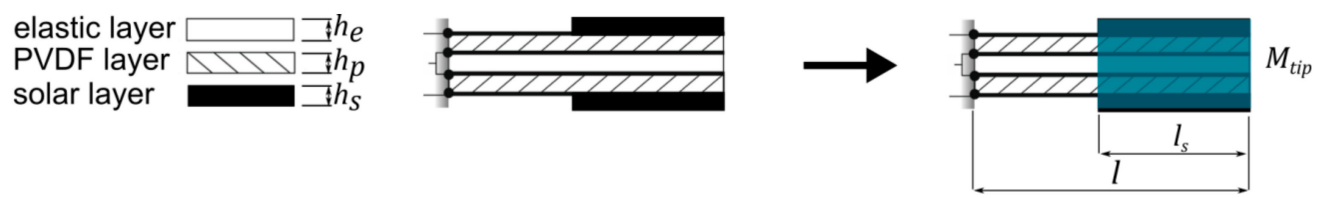

Figure 7. Second modelling approach used in the current study where solar panels are represented as a distributed tip mass. Tip mass is highlighted in blue.

The model developed here is similar to that proposed by Kim and Kim [27] in dealing with distributed masses; however, we extended their model to adopt the new configuration for the harvesters considered in this study. For our arrangement (shown in Figure 7), the tip mass, $M_{\text {tip }}$ is evaluated by:

$$
M_{\text {tip }}=\left(2 \rho_{s} h_{s}+2 \rho_{p} h_{p}+\rho_{e} h_{e}\right) b l_{s}
$$


and the mass moment of inertia around the tip, $I_{\text {tip }}$ is evaluated as:

$$
I_{\text {tip }}=\frac{1}{12} M_{t i p} l_{s}^{2}+2 \rho_{s} h_{s} b l_{s}\left(\left(\frac{l_{s}}{2}\right)^{2}+\left(\frac{h_{e}}{2}+h_{p}+\frac{h_{s}}{2}\right)^{2}\right)+2 \rho_{p} h_{p} b l_{s}\left(\left(\frac{l_{s}}{2}\right)^{2}+\left(\frac{h_{e}}{2}+\frac{h_{p}}{2}\right)^{2}\right)+\rho_{e} h_{e} b l_{s}\left(\frac{l_{s}}{2}\right)^{2}
$$

Note that within this modelling approach, the beam length (i.e., tip location) within the distributed parameter model is equal to $l-l_{s}$. It is useful to define the following two non-dimensional parameters:

$$
\begin{gathered}
\mu=\frac{M_{\text {tip }}}{\rho A_{\text {harv }}\left(l-l_{s}\right)} \\
\gamma=\frac{I_{\text {tip }}}{\rho A_{\text {harv }}\left(l-l_{s}\right)^{3}}
\end{gathered}
$$
by [27]:

The characteristic equation for an Euler-Bernoulli beam with distributed tip mass is thus given

$$
\begin{aligned}
1+\cos \lambda_{n} \cosh \lambda_{n} & +\lambda_{n} \mu\left(\cos \lambda_{n} \sinh \lambda_{n}-\sin \lambda_{n} \cosh \lambda_{n}\right) \\
& -\lambda_{n}^{3} \gamma\left(\cosh \lambda_{n} \sin \lambda_{n}+\sinh \lambda_{n} \cos \lambda_{n}\right)+\lambda_{n}^{4} \mu \gamma\left(1-\cos \lambda_{n} \cosh \lambda_{n}\right) \\
& -\lambda_{n}^{2} \mu\left(\frac{l_{s}}{l-l_{s}}\right) \sinh \lambda_{n} \sin \lambda_{n}-\frac{1}{4} \lambda_{n}^{4} \mu^{2}\left(\frac{l_{s}}{l-l_{s}}\right)^{2}\left(1-\cos \lambda_{n} \cosh \lambda_{n}\right)=0
\end{aligned}
$$

The eigen function can be obtained as [27]:

$$
X_{n}(x)=A_{n}\left[\cosh \lambda_{n} \frac{x}{l-l_{s}}-\cos \lambda_{n} \frac{x}{l-l_{s}}-\sigma_{n}\left[\sinh \lambda_{n} \frac{x}{l-l_{s}}-\sin \lambda_{n} \frac{x}{l-l_{s}}\right]\right]
$$

where $\sigma_{n}$ is obtained from:

$$
\sigma_{n}=\frac{\sinh \lambda_{n}-\sin \lambda_{n}+\lambda_{n} \mu\left(\cosh \lambda_{n}-\cos \lambda_{n}+\frac{\lambda_{n}}{2}\left(\frac{l_{s}}{l-l_{s}}\right)\left(\sinh \lambda_{n}+\sin \lambda_{n}\right)\right)}{\cosh \lambda_{n}+\cos \lambda_{n}+\lambda_{n} \mu\left(\sinh \lambda_{n}-\sin \lambda_{n}+\frac{\lambda_{n}}{2}\left(\frac{l_{s}}{l-l_{s}}\right)\left(\cosh \lambda_{n}-\cos \lambda_{n}\right)\right)}
$$

and the modal amplitude, $A_{n}$, is obtained from a corresponding orthogonality condition:

$$
\begin{aligned}
\int_{0}^{l-l_{s}} X_{s}(x) b\left(\rho_{e} h_{e}\right. & \left.+2 \rho_{p} h_{p}\right) X_{r}(x) d x+X_{s}\left(l-l_{s}\right) M_{t i p} X_{r}\left(l-l_{s}\right) \\
& +\left.M_{t i p} X_{s}\left(l-l_{s}\right) \frac{l_{s}}{2} \frac{d X_{r}(x)}{d x}\right|_{x=l-l_{s}}+\left.M_{t i p} X_{r}\left(l-l_{s}\right) \frac{l_{s}}{2} \frac{d X_{s}(x)}{d x}\right|_{x=l-l_{s}} \\
& +\left.\left.I_{t i p} \frac{d X_{s}(x)}{d x}\right|_{x=l-l_{s}} \frac{d X_{r}(x)}{d x}\right|_{x=l-l_{s}}=\delta_{r s}
\end{aligned}
$$

where $\delta_{r s}$ is the Kronecker delta. The displacement of the harvester at $x=l-l_{s}$ around the first mode is thus obtained from:

$$
w\left(l-l_{s}, t\right)=\psi_{1} X_{1}\left(l-l_{s}\right) \frac{1}{\omega_{1}^{2} \sqrt{\left(1-\left(\frac{\omega}{\omega_{1}}\right)^{2}\right)^{2}+\left(2 \zeta_{1} \frac{\omega}{\omega_{1}}\right)^{2}}} A_{b} \cos \omega t
$$

where the expression for $\psi_{1}$ here is given by [27]:

$$
\psi_{1}=b\left(\rho_{e} h_{e}+2 \rho_{p} h_{p}\right) \int_{0}^{l-l_{s}} X_{1}(x) d x+M_{\text {tip }} X_{1}\left(l-l_{s}\right)+\left.M_{\text {tip }} \frac{l_{s}}{2} \frac{d X_{1}(x)}{d x}\right|_{x=l-l_{s}}
$$


Knowing the displacement distribution in the region $0 \leq x \leq l-l_{s}$ allows to define the angle of the harvester with the horizontal at $x=l-l_{s}$. This angle is denoted as $\theta$ which once evaluated could be used to calculate the displacement of the harvester at $x=l$ :

$$
w(l, t)=w\left(l-l_{s}, t\right)+l_{s} \sin \theta
$$

This means that the harvester is straight within the region $l-l_{s}<x \leq l$.

The parallel connection steady state voltage response, $v_{p}$ for the distributed tip mass model could also be evaluated based on Equation (14) which when operating at the first resonance frequency takes the form:

$$
v_{p, \max }=\left|\frac{j\left(\omega_{1} R_{o p t} \kappa_{1}\right)}{\left(1+j \omega_{1} R_{o p t} C_{p}\right) X_{1}\left(l-l_{s}\right)}\right| w\left(l-l_{s}\right)
$$

where $C_{p}$ is the internal capacitance given by Equation (15) and the modal coupling term, $\kappa_{1}$, for the distributed tip mass model is given by:

$$
\kappa_{1}=2 \frac{d_{31} Y_{p} b_{p}}{2}\left(h_{p}+h_{e}\right) \int_{0}^{l} \frac{d^{2} X_{1}(x)}{d x^{2}} d x=2 \frac{d_{31} Y_{p} b_{p}}{2}\left(h_{p}+h_{e}\right) \int_{0}^{l-l_{s}} \frac{d^{2} X_{1}(x)}{d x^{2}} d x
$$

where the mode shape/eigen function in the above expressions is evaluated based on Equation (24). Note that here $\kappa_{n}$ is only evaluated over the region $0 \leq x \leq l-l_{s}$ as beyond this region the second derivative in the above expression vanishes. This implies that the rigidity of the beam underneath the solar panels prevents any electric power generation from this part of the PVDF layers. Consequently, PVDF power generation from this model is expected to be lower than that from the equivalent concentrated tip mass model which allows the whole beam length to deform and contribute to the power generation. Once the voltage is evaluated, the root mean square value of the peak power could be evaluated from Equation (18).

\subsection{Flexible vs. Rigid Solar Panels}

Figure 8 provides a comparison between the predictions from the two modelling approaches employed in this study for the harvesters with solar panels (equivalent concentrated tip mass model for flexible solar panels and distributed tip mass model for rigid solar panels). The comparison of the tip displacements in Figure 8a indicates that both model results are close, with the rigid tip mass model predictions reaching slightly higher values. The increase in the peak tip displacement predicted from the rigid tip mass model is similar for all harvesters with a value of $6.6 \%$. The differences between the two configurations become more pronounced when comparing the power results, Figure $8 \mathrm{~b}$. As expected the rigid tip mass model predicts lower power values than the flexible tip mass model, on account of the restricted region which is free to deform. However, the penalty due to restricted deformation at the tip is notably small. Comparing peak PVDF power results from both models, it was found that the reduction due to the rigid tip mass was $28 \%$ in cases $\mathrm{H} 5$ and $\mathrm{H} 7$ (shorter harvesters) and $18.5 \%$ in cases $\mathrm{H} 6$ and $\mathrm{H} 8$ (longer harvesters). For a fixed length of solar panel, the loss in performance for the longer harvesters was lower as in these cases a longer portion of PVDF remains uncovered by the solar panels and thus free to deform. 
(a)

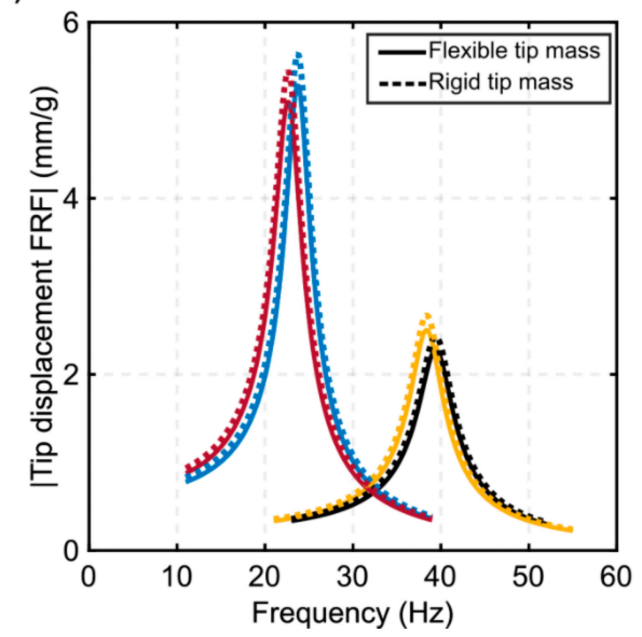

(b)

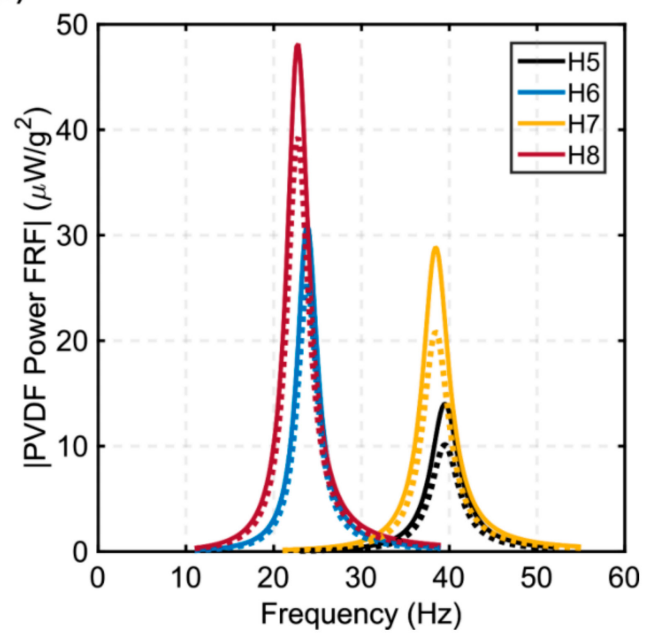

Figure 8. Flexible vs. rigid tip mass model predictions for harvesters with solar panels. (a) Tip displacement frequency response function. (b) PVDF power frequency response function. Solid lines represent flexible tip mass model whereas dashed lines represent rigid tip mass model.

\section{Optimum Tip Mass Configuration}

The developed theoretical models could be used to understand the effect of varying the solar panels configuration on the generated PVDF peak power (i.e., power value at resonance), Figure 9. Note that, in this demonstration, the beam rigidity is evaluated based on the expression for a composite beam in a bimorph configuration [32]. The $x$-axis in Figure 9 represents the solar panels coverage (i.e., $\left.l_{s} / l\right)$ whereas the y-axis represents the ratio of the mass per unit length of the solar panels to the mass per unit length of the harvester (see Equations (1) and (3)), hence providing an indication of the solar panels relative weight. The contours shown in Figure 9 represent the peak power normalized by the maximum peak power value of both plots.

(a)

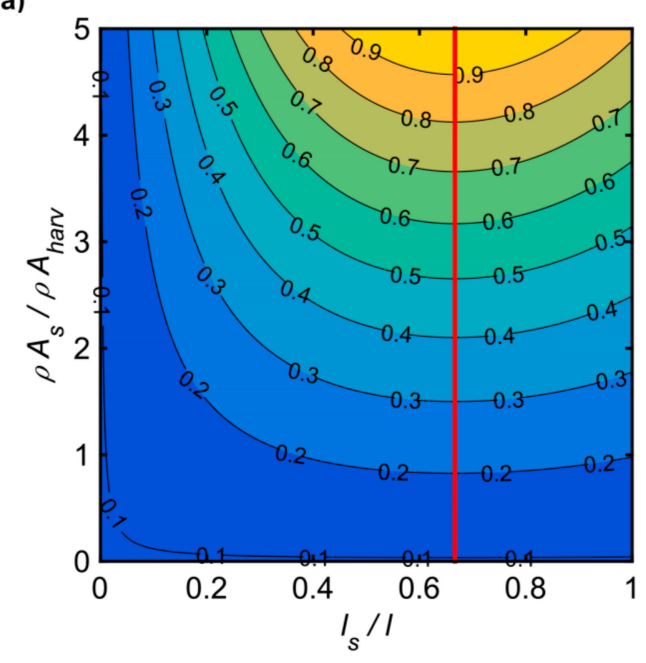

(b)

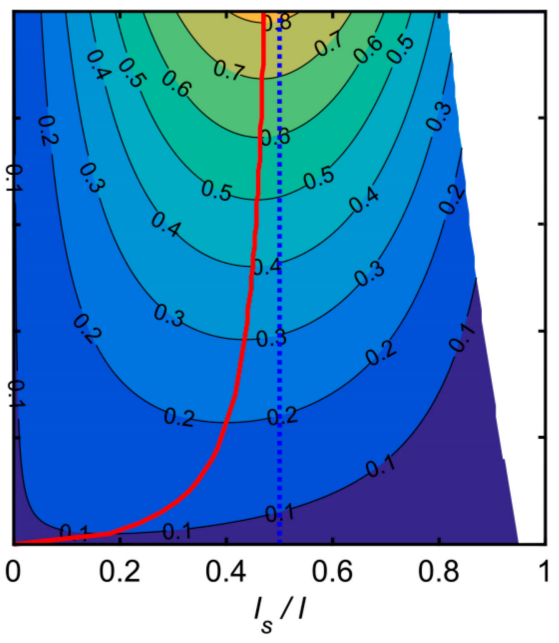

Figure 9. Effect of varying the configuration of solar panels on normalized PVDF peak power output. (a) Equivalent concentrated tip mass model. (b) Distributed tip mass model. Red line represents locus of maximum PVDF peak power for a given tip mass ratio. Blue dashed line in (b) represents an asymptote for maximum PVDF peak power as tip mass ratio increases significantly. Normalization is achieved through dividing contour values by the maximum value of both plots. 
Figure 9 shows that for both models increasing the tip mass ratio will improve the power output. Results for the optimum solar panels coverage (i.e., $l_{s} / l$ ) are more interesting and differ depending on the model employed. For the equivalent concentrated tip mass model, the optimum coverage does not depend on the tip mass ratio and has a constant value of two thirds of the harvester length. This result is expected since if Equation (1) is differentiated with respect to coverage and equated to zero, the resulting quadratic equation has only one feasible root of $2 / 3$. On the other hand, the optimum coverage from the distributed tip mass model is function of the tip mass ratio: it starts at zero for zero tip mass and increases with the increase of the tip mass ratio. Remarkably, it has an asymptotic value of 0.5 for significantly high tip mass ratios. Hence, values for optimum coverage from the distributed tip mass model are always lower than the optimum coverage from the equivalent concentrated tip mass model. Note that Figure $9 \mathrm{~b}$ shows that for high values of coverage, the distributed tip mass model could not provide a solution (white area in Figure 9b) which is a limitation of the model. However, this happens only for values of coverage and tip mass ratio that are far from optimum or practical configurations of interest, hence this model limitation is considered insignificant. As a final note, it would be useful in the future to experimentally test the optimum coverage results predicted. However, this requires customization of the components, since standard available variants are available in limited range of sizes and properties. This goes beyond the scope of the present investigation, and will therefore be addressed in future studies.

\section{Solar Power}

The previous sections showed how solar panels can act as an effective tip mass to affect the performance of the harvesters through decreasing the operational frequency and increasing the PVDF peak power. Whilst these are useful outcomes consistent with their role as a tip mass, a major contribution from the solar panels in terms of power characteristics could be probably attributed to the fact that these panels provide another source of harvesting energy from available light sources. Figure 10 shows the solar power values measured experimentally for the two models of solar panels tested in this study when employing the optimum resistance values identified in Section 2.2. Note that the solar power values shown in Figure 10 are for only one panel (in the present experimental setup, only one solar panel could be illuminated); hence with two solar panels attached to each harvester the power will theoretically be doubled if subjected to the same light exposure. It is important to mention that the power generated from the solar panels is a DC power; however, due to the harvester motion this could change dynamically. Nevertheless, because the tip displacements experienced in this study are relatively low, the effect of the motion from the harvester was negligible as it hardly affected the degree to which the solar panel is incident to the light source, hence an almost constant power was still generated.

The solar power trends shown in Figure 10 were found to follow a near linear variation (at least within the range of Lux measured here). As such, fitting relations were produced for both models and are shown in Figure 10. It could be seen that the power generated from the larger panel (MP3-37) is 1.5 times more than the smaller panel (SP3-37). Nevertheless, if power density is concerned, the larger panel is 1.75 larger in volume, so the smaller panel is more favorable from a power density point of view. It may be useful to note that the lux level for full daylight is around 10,000 lux, for overcast day is around 1000 lux and for a very dark day is around 100 lux. This helps when assessing the expected amount of power generation under various lighting conditions. As such, for a typical overcast condition the SP3-37 should produce $600 \mu \mathrm{W}$ per panel whereas the MP3-37 would produce $900 \mu \mathrm{W}$ per panel. This is much higher magnitude compared to the power obtained from the PVDF elements hence allowing a significant boost to the total power generation from the device. 


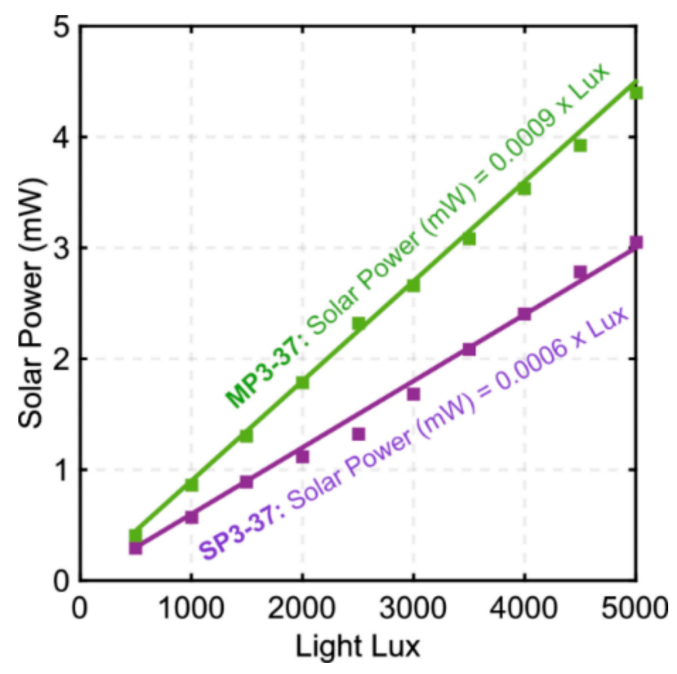

Figure 10. Experimentally measured power from a single solar panel in milli-Watt versus light lux. Markers represent the experimentally measured values whereas lines represent curve fitting for the measured data.

It should be noted that the solar panels employed in this study can in principle be expected to achieve better performance in outdoor applications, where the lighting conditions (intensity and wavelength spectrum) would be more favorable with respect to the indoor LED light illumination considered here. The performance of solar panels in indoor applications is known to depend on the light source used [35,36]. Minnaert and Veelaert [35] showed that LED light illumination, as compared with other light sources, leads to low performance for photovoltaics, with a decrease in performance ranging from a quarter for amorphous silicon up to two thirds for crystalline silicon cells. They also indicate that, under LED light illumination, amorphous silicon cells should perform significantly better with respect to other photovoltaics. The solar panels used here are thin-film amorphous silicon photovoltaics, and should therefore perform reasonably well under LED light illumination. As a matter of fact, according to the solar panel manufacturer (PowerFilm), amorphous silicon has its peak quantum efficiency within a range of wavelengths that matches well the range of wavelengths emitted by LED lights.

Finally, it is worth highlighting that despite the relatively high power output from the solar panels, these panels would have their own limitations when harvesting energy within more realistic conditions. For example, operating in dirty environments could cause the panel to become obscured, and hence affect the amount of energy harvested. The intermittent nature of most light sources, notably daylight, is clearly another limitation. A possible solution would be to integrate the solar panels with energy storage devices, such as batteries and supercapacitors, and use the stored energy when needed.

\section{Conclusions}

This paper shows the effective use of solar panels as active energy harvesting tip masses for PVDF-based vibration energy harvesters suitable for low frequency applications. A total of eight PVDF energy harvesters have been realized, allowing a repetitive assessment of the role of solar panels, together with providing preliminary insights into the effect of changing the length and width on the harvesters' performance. The dynamics of the harvesters was assessed through measuring the frequency response of the tip displacement whereas the PVDF power generation was assessed through measuring the power frequency response. Peak power generation from the solar panels employed in this work over a range of practical light illuminations was also measured and reported. Two electromechanical distributed-parameter models have been developed. The first models the solar panels as an equivalent concentrated tip mass and is more representative of thin, flexible solar panels. Indeed, the model demonstrated good predictive capabilities vs. measured dynamics and PVDF power 
generation responses from the harvesters tested in this work with flexible solar panels. The second model represented the solar panels as rigid distributed tip masses and was developed to investigate the potential loss of power by restricting tip motion, since for up-scaling, larger solar panels may be rigid.

The experimental results obtained confirmed the ability of the solar panels employed in this study to modulate the operation frequency and increase the power generation from the PVDF elements. Considering the planform geometry of the harvesters, PVDF power generation was maximized for the longest and widest harvester, with the length being more influential for performance enhancement. Moreover, solar panels are capable of generating additional harvested power from ambient lighting that can significantly boost the total power generation from the harvester. The theoretical models employed were instrumental in exploring how tip mass configurations could be optimized to improve harvesting performance. In terms of the optimum length of the solar panels, the equivalent concentrated tip mass model showed that having flexible solar panels that are two thirds of the total length will maximize the power output irrespective to the tip mass ratio. The distributed tip mass model, on the other hand, showed that the optimal length for rigid solar panels increases as the tip mass ratio increases, approaching a value that is half of the total length for significantly high tip mass ratios.

Author Contributions: Conceptualization, J.W., M.R.A.N., A.C. and A.R.; Methodology, J.W., M.R.A.N, A.C. and A.R.; Software, M.R.A.N; Validation, J.W and M.R.A.N.; Formal analysis, M.R.AN.; Investigation, J.W., M.R.A.N, A.C. and A.R.; Resources, M.R.A.N., A.C. and A.R.; Data curation, J.W and M.R.A.N.; Writing-original draft preparation, M.R.A.N.; Writing—review and editing, J.W., M.R.A.N, A.C. and A.R.; Visualization, M.R.A.N.; Supervision, M.R.A.N, A.C. and A.R.; Project administration, M.R.A.N, A.C. and A.R.

Funding: This research received no external funding.

Acknowledgments: The authors would like to thank Samuel Weigert from the Department of MACE - The University of Manchester for his generous help in setting up the experimental apparatus in this work. The authors are also grateful to Andrew Kennaugh, Tunde Oyadiji, Steve Burley from the Department of MACE The University of Manchester for their useful contributions during the development of the experimental part of this work.

Conflicts of Interest: The authors declare no conflict of interest.

\section{References}

1. Erturk, A.; Inman, D.J. A distributed parameter electromechanical model for cantilevered piezoelectric energy harvesters. J. Vib. Acoust. 2008, 130, 041002. [CrossRef]

2. Erturk, A.; Inman, D.J. An experimentally validated bimorph cantilever model for piezoelectric energy harvesting from base excitations. Smart Mater. Struct. 2009, 18, 025009. [CrossRef]

3. Renno, J.M.; Daqaq, M.F.; Inman, D.J. On the optimal energy harvesting from a vibration source. J. Sound Vib. 2009, 320, 386-405. [CrossRef]

4. Stanton, S.C.; Erturk, A.; Mann, B.P.; Inman, D.J. Nonlinear piezoelectricity in electroelastic energy harvesters: Modeling and experimental identification. J. Appl. Phys. 2010, 108, 074903. [CrossRef]

5. Abdelkefi, A.; Najar, F.; Nayfeh, A.H.; Ayed, S.B. An energy harvester using piezoelectric cantilever beams undergoing coupled bending-torsion vibrations. Smart Mater. Struct. 2011, 20, 115007. [CrossRef]

6. Orrego, S.; Shoele, K.; Ruas, A.; Doran, K.; Caggiano, B.; Mittal, R.; Kang, S.H. Harvesting ambient wind energy with an inverted piezoelectric flag. Appl. Energy 2017, 194, 212-222. [CrossRef]

7. Li, S.; Lipson, H. Vertical-stalk flapping-leaf generator for wind energy harvesting. In Proceedings of the ASME 2009 Conference on Smart Materials, Adaptive Structures and Intelligent Systems, Oxnard, CA, USA, 21-23 September 2009.

8. Li, S.; Yuan, J.; Lipson, H. Ambient wind energy harvesting using cross-flow fluttering. J. Appl. Phys. 2011, 109, 026104. [CrossRef]

9. Nabawy, M.R.A.; Silva-Leon, J.; O'Connor, J.; Kennaugh, A.; Cioncolini, A.; Revell, A. Simultaneous energy harvesting using dual piezo-solar devices. In Proceedings of the IUTAM Symposium on Critical Flow Dynamics Involving Moving/Deformable Structures with Design Applications, Santorini, Greece, 18-22 June 2018.

10. Silva-Leon, J.; Cioncolini, A.; Nabawy, M.R.A.; Revell, A.; Kennaugh, A. Simultaneous wind and solar energy harvesting with inverted flags. Appl. Energy 2019, 239, 846-858. [CrossRef] 
11. Cioncolini, A.; Nabawy, M.R.A.; Silva-Leon, J.; O'Connor, J.; Revell, A. An experimental and computational study on inverted flag dynamics for simultaneous wind-solar energy harvesting. Fluids 2019, 4, 87. [CrossRef]

12. Vatansever, D.; Hadimani, R.L.; Shah, T.; Siores, E. An investigation of energy harvesting from renewable sources with PVDF and PZT. Smart Mater. Struct. 2011, 20, 055019. [CrossRef]

13. Hobeck, J.D.; Inman, D.J. Artificial piezoelectric grass for energy harvesting from turbulence-induced vibration. Smart Mater. Struct. 2012, 21, 105024. [CrossRef]

14. Song, J.; Zhao, G.; Li, B.; Wang, J. Design optimization of PVDF-based piezoelectric energy harvesters. Heliyon 2017, 3, e00377. [CrossRef] [PubMed]

15. Jiang, Y.; Shiono, S.; Hamada, H.; Fujita, T.; Higuchi, K.; Maenaka, K. Low-frequency energy harvesting using a laminated PVDF cantilever with a magnetic mass. In Proceedings of the Power MEMS 2010: The 10th International Workshop on Micro and Nanotechnology for Power Generation and Energy Conversion Applications, Leuven, Belgium, 30 November-3 December 2010; pp. 375-378.

16. Cao, Z.; Zhang, J.; Kuwano, H. Vibration energy harvesting characterization of $1 \mathrm{~cm}^{2}$ Poly (vinylidene fluoride) generators in vacuum. Jpn. J. Appl. Phys. 2011, 50, 09ND15. [CrossRef]

17. Rammohan, S.; Ramya, C.M.; Kumar, S.J.; Jain, A.; Pratap, R. Low frequency vibration energy harvesting using arrays of PVDF piezoelectric bimorphs. J. Inst. Smart Struct. Syst. 2014, 3, 18-27.

18. Tsukamoto, T.; Umino, Y.; Shiomi, S.; Yamada, K.; Suzuki, T. Bimorph piezoelectric vibration energy harvester with flexible 3D meshed-core structure for low frequency vibration. Sci. Technol. Adv. Mater. 2018, 19, 660-668. [CrossRef] [PubMed]

19. Chandwani, J.; Somkuwar, R.; Deshmukh, R. Multi-band piezoelectric vibration energy harvester for low-frequency applications. Microsyst. Technol. 2019, 25, 3867-3877. [CrossRef]

20. Jacobson, M.Z.; Delucchi, M.A. A path to sustainable energy by 2030. Sci. Am. 2009, 301, 58-65. [CrossRef] [PubMed]

21. Haji, M.N.; Kluger, J.M.; Sapsis, T.P.; Slocum, A.H. A symbiotic approach to the design of offshore wind turbines with other energy harvesting systems. Ocean Eng. 2018, 169, 673-681. [CrossRef]

22. Iqbal, M.; Khan, F.U. Hybrid vibration and wind energy harvesting using combined piezoelectric and electromagnetic conversion for bridge health monitoring applications. Energy Convers. Manag. 2018, 172, 611-618. [CrossRef]

23. Gambier, P.; Anton, S.R.; Kong, N.; Erturk, A.; Inman, D.J. Piezoelectric, solar and thermal energy harvesting for hybrid low-power generator systems with thin-film batteries. Meas. Sci. Technol. 2011, 23, 015101. [CrossRef]

24. Rao, S.S. Mechanical Vibrations, 2nd ed.; Adison-Wesley Publishing Company: New York, NY, USA, 1990; pp. 22-23.

25. Roundy, S.; Wright, P.K. A piezoelectric vibration based generator for wireless electronics. Smart Mater. Struct. 2004, 13, 1131. [CrossRef]

26. Kim, M.; Hoegen, M.; Dugundji, J.; Wardle, B.L. Modeling and experimental verification of proof mass effects on vibration energy harvester performance. Smart Mater. Struct. 2010, 19, 045023. [CrossRef]

27. Kim, J.E.; Kim, Y.Y. Analysis of piezoelectric energy harvesters of a moderate aspect ratio with a distributed tip mass. J. Vib. Acoust. 2011, 133, 041010. [CrossRef]

28. Strutt, J.W. (Lord Rayleigh). The Theory of Sound; Macmillan: London, UK, 1877.

29. Roundy, S.; Wright, P.K.; Rabaey, J. A study of low level vibrations as a power source for wireless sensor nodes. Comput. Commun. 2003, 26, 1131-1144. [CrossRef]

30. Park, J.; Lee, S.; Kwak, B.M. Design optimization of piezoelectric energy harvester subject to tip excitation. J. Mech. Sci. Technol. 2012, 26, 137-143. [CrossRef]

31. Erturk, A.; Inman, D.J. Piezoelectric Energy Harvesting; John Wiley \& Sons: West Sussex, UK, 2011; pp. 19-130.

32. Nabawy, M.R.A.; Crowther, W.J. Dynamic electromechanical coupling of piezoelectric bending actuators. Micromachines 2016, 7, 12. [CrossRef] [PubMed]

33. Nabawy, M.R.A.; Parslew, B.; Crowther, W.J. Dynamic performance of unimorph piezoelectric bending actuators. Proc. Inst. Mech. Eng. Part I J. Syst. Control Eng. 2015, 229, 118-129. [CrossRef] 
34. Aldraihem, O.J.; Wetherhold, R.C.; Singh, T. Distributed control of laminated beams: Timoshenko theory vs. Euler-Bernoulli theory. J. Intell. Mater. Syst. Struct. 1997, 8, 149-157. [CrossRef]

35. Minnaert, B.; Veelaert, P. Which type of solar cell is best for low power indoor devices. In Innovation for Sustainable Production: i-SUP 2010; Ghent University, Department of Electronics and Information Systems: Ghent, Belgium, 2010.

36. Minnaert, B.; Veelaert, P. A proposal for typical artificial light sources for the characterization of indoor photovoltaic applications. Energies 2014, 7, 1500-1516. [CrossRef]

(C) 2019 by the authors. Licensee MDPI, Basel, Switzerland. This article is an open access article distributed under the terms and conditions of the Creative Commons Attribution (CC BY) license (http://creativecommons.org/licenses/by/4.0/). 